\title{
Exploiting quantitative trait loci in gene discovery
}

\author{
Sarah Hake ${ }^{1,3}$ and Torbert Rocheford ${ }^{2}$ \\ ${ }^{1}$ Plant Gene Expression Center, U.S. Department of Agriculture-Agricultural Research Science, University of California, \\ Berkeley, Albany, California 94710, USA; ${ }^{2}$ Department of Crop Sciences, University of Illinois, Urbana, Illinois 61801, USA
}

Developmental biologists prefer to study single gene mutations that produce large obvious changes, changes that may be accompanied by lethality or sterility. Such phenotypes are easy to find in genetic screens and reveal that the gene is required for early, fundamental processes, and thus is important for the organism. Natural variation, however, does not work that way; mutations that lead to sterility or embryonic lethality are quickly weeded out. The alleles selected by nature contribute to survival of the organism, not its detriment. In addition, the variation between two different, naturally occurring populations is not likely to be due to a single gene, but multiple allelic differences. A similar contrast exists when we consider breeding for agricultural purposes versus genetic studies in the laboratory. Breeders select for a consistent phenotype, and often the traits they select for are subtle, assayed quantitatively in large segregating populations. The traits are usually the result of many gene differences, not single Mendelian mutations.

How do we find the genes that distinguish one population from another, or that distinguish a wild from domesticated species? Plants are particularly useful for such studies, as crossing barriers are often geographic, and thus, the experimenter can create F2 progeny that will segregate for the components of the two parental phenotypes. When the F2 individuals do not fall into discrete classes due to segregation at one or two genes, the trait is considered to be quantitatively inherited, and the loci that contribute to the differences between the parents are referred to as quantitative trait loci (QTL).

\section{Identifying alleles responsible for natural variation}

The use of naturally occurring variation for gene identity in Arabidopsis has been championed by Maartin Koornneef and his colleagues (Alonso-Blanco and Koornneef 2000). Arabidopsis is a self-pollinated weed that grows in varied ecological niches, thus different accessions often

${ }^{3}$ Corresponding author.

E-MAIL maizesh@nature.berkeley.edu; FAX (510) 559-5678.

Article and publication are at http://www.genesdev.org/cgi/doi/10.1101/ gad.1199604 show a range of phenotypes and growth responses. To determine the number of genes responsible for observed traits and to map their position, recombinant inbred populations have been developed. Recombinant inbred (RI) lines have been used extensively for crop plants and also exploited in Arabidopsis. RI lines are created by self-pollinating an F2 population for six or more generations, thus creating highly homozygous lines that differ from each other (Burr et al. 1988). Because each RI line is theoretically nearly homozygous at every locus, the variation among the lines is immortalized, and the RI lines can be propagated and multiplied. A set of probes that cover the genome and distinguish alleles from the two parents provides map positions for the genes controlling the traits that are analyzed. The resolution of the map position is largely a function of the number of RI lines and assayed probes for the population.

The beauty of an RI population is that it can be screened for different traits by different people and at different times. Interestingly, often the variation in the RI lines extends beyond the variation seen in the original parents. This phenomenon, called transgressive segregation, is the basis of many breeding and selection regimes, and has been characterized in QTL (deVicente and Tanksley 1993). Transgressive segregation is due to the creation of new combinations of alleles at multiple loci relative to each of the two parents. For example, if a gene that promotes root growth is expressed in the same parent as a gene that inhibits root growth, the roots would likely be of average length. Progeny derived from a cross starting with this line, on the other hand, could have longer or shorter roots, depending on recombination with the alleles of the other contributing parent.

One of the RI populations studied in Arabidopsis was made by Koornneef's group using the Cape Verde Islands (Cvi) accession and the Landsberg erecta (Ler) laboratory strain (Alonso-Blanco et al. 1998). The Cvi/Ler population has been examined for flowering time (AlonsoBlanco et al. 1998), seed dormancy (Alonso-Blanco et al. 2003), and circadian rhythms (Swarup et al. 1999), among other traits. The population was also grown in seven different light and hormone environments (Borevitz et al. 2002). Transgressive segregation was identified in 
each environment, notably with phenotypes close to that of loss-of-function mutations. In this particular experiment, 12 QTL were identified, some of which mapped to known genes, such as PHYTOCHROME $B$ and others that mapped to no previously identified loci in regard to hormone or light responses.

\section{Identifying $B R X$, a naturally occurring allele that regulates root length}

Mouchel et al. (2004) set out to find previously unidentified genes that regulate root-system morphology. They examined primary root length of 44 isogenic accessions of Arabidopsis (Alonso-Blanco and Koornneef 2000), growing them in the same controlled conditions. Root length varied sixfold, with the shortest roots belonging to the Unkirch (Uk-1) accession. Crosses of Uk-1 with Slavice-O (Sav-O), an accession with average roots, revealed that the short-root phenotype behaved as a recessive in the F2 generation, with ratios near the expectations of a single gene. The locus was named BREVIS $R A D I X(B R X)$.

RI lines were developed using Sav-O and Uk-1 parents and analyzed for root length. The genotype of each line was determined for a set of 17 simple sequence length polymorphisms across the genome. The analysis identified a major contributing locus on chromosome 1. Segregation for this QTL explained $80 \%$ of the phenotypic variation for root length in this RI population.

To create a near isogenic line, the F2 individual with the shortest root was crossed to Sav-O, then self-pollinated. The new individual with the shortest root was again crossed to Sav-O and self-pollinated. After four rounds of backcrossing and selfing, the short-root line was considered to have $\sim 97 \%$ of the Sav-O genome and only $3 \%$ of the Uk-1 genome. The roots of the near isogenic line, which they named $b r x^{s}$, were almost as short as the Uk-1 roots. Other phenotypic differences between Uk-1 and Sav-O, such as flowering time and number of axillary branches, disappeared, as the genomic regions controlling these traits became homozygous for Sav-O alleles.

The $b r x^{s}$ genotype provided an opportunity to make detailed comparisons with Sav-O with regard to cell size and organization in the root. Their analysis suggested that $b r x^{s}$ primarily regulates cell division. Both the meristematic and elongation zone of the root were affected, although the phenotypic differences were most pronounced in the meristematic zone. Because the shortroot phenotype was followed in their crossing scheme, rather than markers linked to the $B R X$ locus, alleles at other loci that contribute in small ways to the short-root phenotype may have also been selected, and thus are responsible for the short-root phenotype.

Most root mutants actually affect components of the shoot as well, for example, the hobbit (Blilou et al. 2002) or scarecrow mutant of Arabidopsis (Wysocka-Diller et al. 2000). By starting with naturally occurring variation and introgressing the QTL into the other accession, Mouchel and colleagues (Mouchel et al. 2004) were able to show that the root phenotype was separable from shoot differences of the two ecotypes. Root phenotypes are often tied to alterations in hormone signaling; for example, roots grown on cytokinin, auxin, or jasmonic acid are shorter than normal. The effect $B R X$ had on root length was not dependent on growth conditions, nor was $b r x^{s}$ defective in hormone signaling or basic physiological responses.

A fine map position for the gene was obtained by examining 860 F2 progeny from the original cross of Sav-O and Uk-1. The power of Arabidopsis came to play with a fully sequenced genome, providing markers as well as candidate genes in the QTL interval defined by recombinants. Of the 10 genes in the $45-\mathrm{kb}$ interval, they sequenced eight in the Uk-1 background. One gene, At1g31880, carried a premature stop codon in Uk-1, but not in the Columbia genome, which was sequenced by the Arabidopsis consortium. The stop codon in Atlg31880 was not found in 12 other accessions, including some that were originally isolated near the vicinity of Uk-1. Transformation of the Columbia allele into the $b_{r x}{ }^{s}$ line gave $80 \%$ of the root length of Sav-O. This result is consistent with their analysis of the recombinant inbred population. One might ask what is the selective advantage of short roots? The Uk-1 accession has been collected from a river embankment (The Arabidopsis Information Resourse, http://www.arabidopsis.org), thus long roots may not be needed due to a higher water table.

The work serves as an important example of the need for multiple approaches to gene discovery and the power of a sequenced genome. The $B R X$ gene would not have been identified by gene-profiling experiments, it is expressed at very low levels and is also expressed in shoots. It is possible that it might have been identified by mutagenesis in the Sav-O background, but it is not known whether it would have surfaced in mutagenesis of the typically used Columbia background. Had the scientists only looked at candidate genes, $B R X$ would not have been identified; it was incorrectly annotated at the time and previously unidentified. Gene discovery often occurs as members of gene families are slowly teased apart using reverse genetics. Again, BRX would have likely eluded their search, as insertions into other gene family members do not have a root phenotype.

\section{Cloning QTL that regulate flowering time}

Recombinant inbred lines have also been successfully exploited to clone some of the QTL that regulate flowering time in Arabidopsis. Most Arabidopsis plants flower earlier in long days than in short days. Cvi and Ler plants are similar in their flowering time, although Cvi is a little less sensitive to day length. When AlonsoBlanco and colleagues (Alonso-Blanco et al. 1998) examined the Cvi/Ler RI lines for flowering time, they found that some lines flowered much earlier or much later than either parent, under both long days and short days. A near isogenic line was created that contained one of the four major QTL, named early day length insensitive 
(EDI), by backcrossing the Cvi allele of EDI into Ler and following the trait by phenotypic and genotypic selection (Alonso-Blanco et al. 1998). The near isogenic line flowered much earlier than Ler in short days.

By following recombination of flanking markers, the group was able to localize EID to a region of the genome spanning 15 ORFs, including one that encoded the bluelight photoreceptor, cryptochrome 2 (El-Din El-Assal et al. 2001). CRY2 was a reasonable candidate gene, as recessive mutations at this locus flower late, especially in long days (Guo et al. 1998). To prove that the QTL was $C R Y 2$, they transformed the Ler CRY2 allele into Cvi and the Cvi allele into Ler. The flowering time was dependent on the CRY2 allele used in the transformation. Comparison of amino acid differences between Cvi, Ler, and other accessions suggested that one amino acid was critical. Sure enough, when they made that change in the Cvi allele, they abolished the early flowering (El-Din ElAssal et al. 2001).

An explanation for transgressive segregation can be seen in this analysis. Cvi alleles at two QTL result in early flowering, whereas Cvi alleles at two other loci create late flowering. Thus, Cvi is similar to Ler, but the RI lines can be quite different from either parent when the right combination of alleles segregates.

Arabidopsis flowering is also regulated by vernalization, which is a long exposure to cold. Some late-flowering accessions need a period of vernalization in order to flower. This strategy keeps them from flowering in the fall in climates where they would freeze in the winter. Both the FRI and FLC loci were first identified as QTL in the vernalization response (Michaels and Amasino 1999; Johanson et al. 2000). FRI was cloned by its position and encodes a novel protein. Most of the early flowering lines, such as those used in laboratories, have deletions in the FRI locus. A survey of 38 different alleles from late and early flowering lines showed that dominant FRI alleles are found in accessions where long periods of cold are required for flowering, whereas recessive alleles harboring one deletion or another, are found in early flowering accessions (Johanson et al. 2000).

Flowering time is also extremely important in crop plants, and QTL that regulate this trait have been identified. Heading date1 (Hd1) is one of four major QTL in rice that controls flowering time in response to photoperiod. Mapping of the QTL placed it to within a 12-kb region (Yano et al. 2000). A homolog to CONSTANS, which functions in the perception of photoperiod in Arabidopsis (Putterill et al. 1995), was found in that interval and proven to be Hd1. Thus, the combination of a small interval and an excellent candidate gene allowed the cloning of this QTL (Yano et al. 2000).

\section{Identifying alleles selected during crop domestication}

Domestication of wild tomatoes into the variety we eat led to a dramatic increase in fruit size. The F2 progeny of a cross between a wild and cultivated tomato show continuous variation in size. The number of QTL range between 11 and 20, depending on the particular population studied, with each QTL contributing from $5 \%$ to $42 \%$ of the phenotypic variance (Paterson et al. 1991). Fruit weight 2.2 (fw2.2) is a QTL that accounts for $5 \%-30 \%$ of the phenotypic variance in an F2 population and up to $47 \%$ when introgressed from the wild species, Lycopersicon pennellii, into cultivated tomato, Lycopersicon esculentum (Alpert et al. 1995). Tanksley and his group were able to identify a YAC that contained this QTL by screening a population of 3500 near-isogenic individuals to identify recombinants in the region flanking the QTL. The recombinants were analyzed for fruit weight using the statistical power of replicated plots and different field locations (Alpert and Tanksley 1996). The group was able to identify the actual gene responsible for the QTL by transformation of cosmids made from L. pennellii DNA into cultivated varieties; the fruit weight of one transgenic line was statistically different from controls (Frary et al. 2000). This tremendous effort of mapping and transformation identified a low-expression gene with structural similarity to a human oncogene. The basis by which the $L$. pennellii allele produces smaller fruit than $L$. esculentum may be due to regulatory differences (Frary et al. 2000).

The domestication of maize, which is thought to have begun $~ 9000$ years ago (Matsuoka et al. 2002), is another example in which strong selective forces have had an impact on allelic diversity. Maize has a single stalk that terminates in a male inflorescence, called a tassel. The female inflorescences, ears, are at the ends of short branches. Teosinte has many branches, all long and ending in tassels. Numerous other differences exist, such as seed shattering and hardness of the seed coat. Despite their morphological differences, the two plants produce fertile F2 progeny, which segregate for the entire range of phenotypes. John Doebley was able to map the differences between maize and teosinte to five loci with major effect. One of these quantitative trait loci mapped to the region of a known maize mutant, teosinte branched1 (tb1; Doebley and Stec 1991).

Doebley introgressed the teosinte QTL into maize, using linked markers as a guide. He was then able to ask whether this stock, mostly maize, but carrying a small segment of the teosinte genome, complemented the maize $t b 1$ recessive mutation (Doebley et al. 1995). The complementation test failed, indicating that the QTL of teosinte was an allele of the $t b 1$ gene. $t b 1$ accounts for some of the architectural differences between maize and teosinte, but not the seed aspects. Because $t b 1$ was defined by a maize mutation, Doebley was able to clone the QTL by transposon tagging tb1 (Doebley et al. 1997). The $t b 1$ gene is expressed in both species, but in different tissues and at different levels (Doebley et al. 1997; Hubbard et al. 2002).

Sequence comparisons of $t b 1$ in a large number of maize inbreds and teosinte accessions showed similar levels of sequence polymorphism across the gene in maize and teosinte, but little diversity in the maize promoter compared with diversity in teosinte $5^{\prime}$ sequences (Wang et al. 1999). These results suggest that domestication acted on the regulation of the $t b 1$ gene. Other 
QTL that are responsible for the difference in seed coat (Dorweiler et al. 1993) remain unknown, as they fall into regions presently lacking candidate genes.

Artificial selection has influenced not only the architecture of the maize plant, but also numerous aspects of seed quality. Ed Buckler has taken advantage of the high level of polymorphism in maize and the large number of defined inbreds to examine different genes for evidence of selection (Flint-Garcia et al. 2003). This strategy, known as association analysis, is a search for a polymorphism that is associated with a particular trait. The candidate gene is sequenced across an established set of inbreds or accessions that are intentionally selected to represent diversity of the species. This collection of inbreds is treated as a mapping population for the species. The enormous number of recombination events that took place since the diverse lines shared common ancestors provides greater confidence that an association is meaningful. A caveat of this approach is the possibility that the sequenced gene is simply linked to an allele under selection. In maize, the nonrandom association of alleles at different loci, also called linkage disequilibrium, falls off rapidly outside of a gene (Flint-Garcia et al. 2003). Thus, the likelihood that an amino acid change is associated with a trait is much higher. Linkage disequilibrium does not fall off rapidly outside of a gene in Arabidopsis, partially because of its self-pollinating nature (Nordborg et al. 2002).

Buckler found that the nucleotide diversity was unusually low at six loci in the starch pathway, whereas the polymorphism is high at random maize loci with an average of $1.3 \%$ nucleotide diversity (Tenaillon et al. 2001). Similarly, nucleotide comparisons at the anthocyanin gene, $c 1$, found very few polymorphisms, suggesting that maize has also undergone selection at this locus (Hanson et al. 1996). Three candidate genes for selection during domestication were also sequenced in ancient corn-cob DNA, and revealed that even 4000 years ago, selection had affected the diversity of alleles in maize (Jaenicke-Despres et al. 2003).

The maize inflorescence provides another unique opportunity to examine genetic diversity that may have been under natural or artificial selection. A number of well-described mutations exist that affect the architecture of the tassel and ear (Veit et al. 1993; McSteen et al. 2000), for example, mutations that affect the length and number of branches. At the same time, examination of tassel traits in different RI populations has identified QTL that regulate inflorescence architecture. Interestingly, for many of the QTL, there is a candidate gene defined by a mutant phenotype (Berke and Rocheford 1999|. Because maize is related to rice and other grasses, the genes identified by QTL or mutation serve as tools to examine the evolution of inflorescence architecture in other grasses, where the diversity of form is rich (Kellogg 2001). The branched silkless1 gene is an example of a gene conserved in sequence, function, and expression in maize and rice, two fairly diverged grass species (Chuck et al. 2002). ramosa1 provides an opposite example, it appears to be missing in rice (E. Vollbrecht and R. Mar- tienssen, pers. comm.) and maps to a QTL in maize (T. Rocheford, unpubl.).

\section{Summary}

Extensive efforts over the past $10 \mathrm{yr}$ have led to the mapping of QTL for a broad array of plant phenotypes in diverse species. The resulting datasets can be compared with map locations of mutants and cloned genes. Depending on the trait and the relevant resources available, different strategies may be pursued to uncover the gene(s) underlying a QTL. As with the cloning of $t b 1$ and $C R Y 2$, there may be a candidate gene previously defined by a null allele. In the case of $B R X, F R I$, and fw2.2, there were no candidate genes, but the ability to create new alleles or use transgenics provided proof that the QTL was cloned. In species with large genomes, a number of candidate genes may reside within a QTL region defined by recombination. In this case, sequencing in a number of diverse inbreds can help find the gene that carries polymorphism significantly associated for the trait. Just as the molecular biologist searches out the important domains or amino acids of a protein, the use of different QTL populations uncovers allelic variation. The availability of complete genome sequence in many plant species will greatly enhance the ability to more rapidly characterize and exploit this allelic variation.

\section{Acknowledgments}

We thank China Lunde for reading a draft of the manuscript; our colleagues on the inflorescence project; R. Martienssen, D. Jackson, E. Kellogg, R. Schmidt, V. Brendel, and E. Vollbrecht; and NSF (DBI-0110189) for funding the project.

\section{References}

Alonso-Blanco, C. and Koornneef, M. 2000. Naturally occurring variation in Arabidopsis: An underexploited resource for plant genetics. Trends Plant Sci. 5: 22-29.

Alonso-Blanco, C., El-Assal, S.E., Coupland, G., and Koornneef, M. 1998. Analysis of natural allelic variation at flowering time loci in the Landsberg erecta and Cape Verde Islands ecotypes of Arabidopsis thaliana. Genetics 149: 749-764.

Alonso-Blanco, C., Bentsink, L., Hanhart, C.J., Blankestijn-de Vries, H., and Koornneef, M. 2003. Analysis of natural allelic variation at seed dormancy loci of Arabidopsis thaliana. Genetics 164: 711-729.

Alpert, K.B. and Tanksley, S.D. 1996. High-resolution mapping and isolation of a yeast artificial chromosome contig containing fw2.2: A major fruit weight quantitative trait locus in tomato. Proc. Natl. Acad. Sci. 93: 15503-15507.

Alpert, K.B., Grandillo, S., and Tanksley, S.D. 1995. fw2.2: A major QTL controlling fruit weight is common to both redand green-fruited tomato species. Theor. Appl. Genet. 91: 994-1000.

Berke, T.G. and Rocheford, T.F. 1999. Quantitative trait loci for tassel traits in maize. Crop Sci. 39: 1439-1443.

Blilou, I., Frugier, F., Folmer, S., Serralbo, O., Willemsen, V., Wolkenfelt, H., Eloy, N.B., Ferreira, P.C., Weisbeek, P., and Scheres, B. 2002. The Arabidopsis HOBBIT gene encodes a 
CDC27 homolog that links the plant cell cycle to progression of cell differentiation. Genes \& Dev. 16: 2566-2575.

Borevitz, J.O., Maloof, J.N., Lutes, J., Dabi, T., Redfern, J.L., Trainer, G.T., Werner, J.D., Asami, T., Berry, C.C., Weigel, D., et al. 2002. Quantitative trait loci controlling light and hormone response in two accessions of Arabidopsis thaliana. Genetics 160: 683-696.

Burr, B., Burr, F.A., Thompson, K.H., Albertson, M.C., and Stuber, C.W. 1988. Gene mapping with recombinant inbreds in maize. Genetics 118: 519-526.

Chuck, G., Muszynski, M., Kellogg, E., Hake, S., and Schmidt, R.J. 2002. The control of spikelet meristem identity by the branched silkless1 gene in maize. Science 298: 1238-1241.

deVicente, M.C. and Tanksley, A.S.D. 1993. QTL Analysis of transgressive segregation in an interspecific tomato cross. Genetics 134: 585-596.

Doebley, J. and Stec, A. 1991. Genetic-analysis of the morphological differences between maize and teosinte. Genetics 129: 285-295.

Doebley, J., Stec, A., and Gustus, C. 1995. teosinte branched1 and the origin of maize: Evidence for epistasis and the evolution of dominance. Genetics 141: 333-346.

Doebley, J., Stec, A., and Hubbard, L. 1997. The evolution of apical dominance in maize. Nature 386: 485-488.

Dorweiler, J., Stec, A., Kermicle, J., and Doebley, J. 1993. Teosinte glume architecture1: A genetic locus controlling a key step in maize evolution. Science 262: 233-235.

El-Din El-Assal, S., Alonso-Blanco, C., Peeters, A.J., Raz, V., and Koornneef, M. 2001. A QTL for flowering time in Arabidopsis reveals a novel allele of CRY2. Nat. Genet. 29: 435-440.

Flint-Garcia, S.A., Thornsberry, J.M., and Buckler, E.S. 2003. Structure of linkage disequilibrium in plants. Annu. Rev. Plant Biol. 54: 357-374.

Frary, A., Nesbitt, T.C., Grandillo, S., Knaap, E., Cong, B., Liu, J., Meller, J., Elber, R., Alpert, K.B., and Tanksley, S.D. 2000. fw2.2: A quantitative trait locus key to the evolution of tomato fruit size. Science 289: 85-88.

Guo, H., Yang, H., Mockler, T.C., and C, L. 1998. Regulation of flowering time by Arabidopsis photoreceptors. Science 279: 1360-1363.

Hanson, M.A., Gaut, B.S., Stec, A.O., Fuerstenberg, S.I., Goodman, M.M., Coe, E.H., and Doebley, J.F. 1996. Evolution of anthocyanin biosynthesis in maize kernels: The role of regulatory and enzymatic loci. Genetics 143: 1395-1407.

Hubbard, L., McSteen, P., Doebley, J., and Hake, S. 2002. Expression patterns and mutant phenotypes of teosinte brached1 correlate with growth suppression in maize and teosinte. Genetics 162: 1927-1935.

Jaenicke-Despres, V., Buckler, E., Smith, B.D.S., Gilbert, M.T.P., Cooper, A., Doebley, J., and Paabo, S. 2003. Early allelic selection in maize as revealed by ancient DNA. Science 302: 1206-1208.

Johanson, U., West, J., Lister, C., Michaels, S., Amasino, R., and Dean, C. 2000. Molecular analysis of FRIGIDA, a major determinant of natural variation in Arabidopsis flowering time. Science 290: 344-347.

Kellogg, E.A. 2001. Evolutionary history of the grasses. Plant Physiol. 125: 1198-1205.

Matsuoka, Y., Vigouroux, V., Goodman, M.M., Sanchez, G.J., Buckler, E., and Doebley, J. 2002. A single domestication for maize shown by multilocus microsatellite genotyping. Proc. Natl. Acad. Sci. 99: 6080-6084.

McSteen, P., Laudencia-Chingcuanco, D., and Colasanti, J. 2000. A floret by any other name: Control of meristem identity in maize. Trends Plant Sci. 5: 61-66.

Michaels, S.D. and Amasino, R. 1999. FLOWERING LOCUS C encodes a novel MADS domain protein that acts as a repressor of flowering. Plant Cell 11: 949-956.

Mouchel, C.F., Briggs, G.C., and Hardtke, C.S. 2004. Natural genetic variation in Arabidopsis identifies BREVIS RADIX, a novel regulator of cell proliferation and elongation in the root. Genes \& Dev. (this issue).

Nordborg, M., Borevitz, J.O., Bergelson, J., Berry, C.C., Chory, J., Hagenblad, J., Kreitman, M., Maloof, J.N., Noyes, T., Oefner, P.J., et al. 2002. The extent of linkage disequilibrium in Arabidopsis thaliana. Nat. Genet. 30: 190-193.

Paterson, A.H., Damon, S., Hewitt, J.D., Zamir, D., Rabinowitch, H.D., Lincoln, S.E., Lander, E.S., and Tanksley, S.D. 1991. Mendelian factors underlying quantitative traits in tomato: Comparison across species, generations, and environments. Genetics 127: 181-197.

Putterill, J., Robson, F., Lee, K., Simon, R., and Coupland, G. 1995. The CONSTANS gene of Arabidopsis promotes flowering and encodes a protein showing similarities to zinc finger transcription factors. Cell 80: 847-857.

Swarup, K., Alonso-Blanco, C., Lynn, J.R., Michaels, S.D., Amasino, R.M., Koornneef, M., and Millar, A.J. 1999. Natural allelic variation identifies new genes in the Arabidopsis circadian system. Plant I. 20: 67-77.

Tenaillon, M.I., Sawkins, M.C., Long, A.D., Gaut, R.L., Doebley, J.F., and Gaut, B.S. 2001. Patterns of DNA sequence polymorphism along chromosome 1 of maize (Zea mays ssp. mays L.). Proc. Nat1. Acad. Sci. 98: 9161-9166.

Veit, B., Schmidt, R.J., Hake, S., and Yanofsky, M.F. 1993. Maize floral development-new genes and old mutants. Plant Cell 5: 1205-1215.

Wang, R.-L., Stec, A., Hey, J., Lukens, L., and Doebley, J. 1999. The limits of selection during maize domestication. Nature 398: 236-239.

Wysocka-Diller, J.W., Helariutta, Y., Fukaki, H., Malamy, J.E., and Benfey, P.N. 2000. Molecular analysis of SCARECROW function reveals a radial patterning mechanism common to root and shoot. Development 127: 595-603.

Yano, M., Katayose, Y., Ashikari, M., Yamanouchi, U., Monna, L., Fuse, T., Baba, T., Yamamoto, K., Umehara, Y., Nagamura, Y., et al. 2000. Hd1, a major photoperiod sensitivity quantitative trait locus in rice, is closely related to the Arabidopsis flowering time gene CONSTANS. Plant Cell 12: $2473-2484$. 


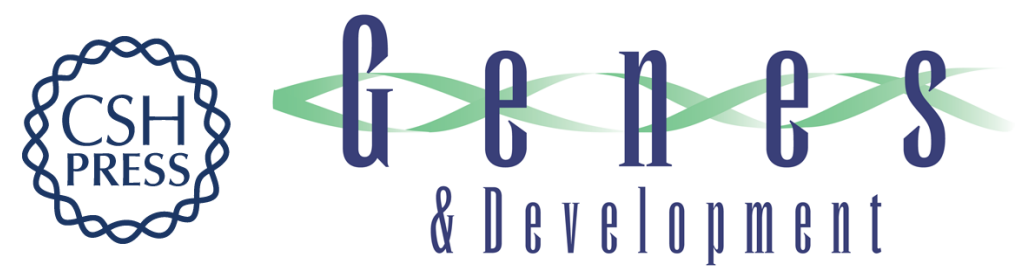

\section{Exploiting quantitative trait loci in gene discovery}

Sarah Hake and Torbert Rocheford

Genes Dev. 2004, 18:

Access the most recent version at doi:10.1101/gad.1199604

References This article cites 36 articles, 25 of which can be accessed free at: http://genesdev.cshlp.org/content/18/6/597.full.html\#ref-list-1

License

Email Alerting Receive free email alerts when new articles cite this article - sign up in the box at the top Service right corner of the article or click here.

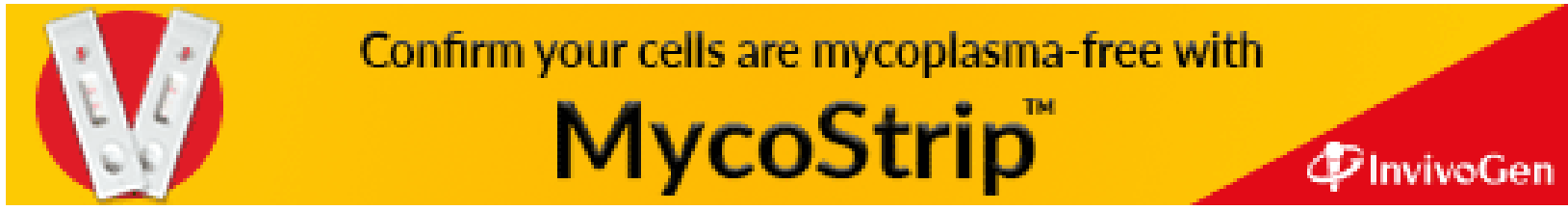

